\title{
L'expérience agricole des citadins dans les jardins collectifs urbains : le cas de Montpellier
}

Urban gardening: reinstate agricultural act in the city

\section{Pascale Scheromm}

\section{(2) OpenEdition}

1 Journals

\section{Édition électronique}

URL : http://journals.openedition.org/developpementdurable/10726

DOI : 10.4000/developpementdurable.10726

ISSN : 1772-9971

Éditeur

Association DD\&T

\section{Référence électronique}

Pascale Scheromm, "L'expérience agricole des citadins dans les jardins collectifs urbains : le cas de Montpellier », Développement durable et territoires [En ligne], Vol.6, n | Mars 2015, mis en ligne le 31 mars 2015, consulté le 19 avril 2019. URL : http://journals.openedition.org/ developpementdurable/10726 ; DOI : 10.4000/developpementdurable.10726

Ce document a été généré automatiquement le 19 avril 2019.

Développement Durable et Territoires est mis à disposition selon les termes de la licence Creative Commons Attribution - Pas d'Utilisation Commerciale 4.0 International. 


\title{
L'expérience agricole des citadins dans les jardins collectifs urbains : le cas de Montpellier
}

Urban gardening: reinstate agricultural act in the city

\author{
Pascale Scheromm
}

Tous mes remerciements à C. Soulard (UMR Innovation) pour ses conseils et son soutien dans l'élaboration de ce travail.

1 La question de la production alimentaire locale dans les villes et à leur proximité connaît ces dernières décennies un véritable regain d'intérêt. Parmi les différentes formes d'agriculture relevant de relocalisation de la production agricole, les jardins collectifs, parcelles ou groupes de parcelles cultivés par un ensemble de jardiniers, sont en plein essor dans les villes des pays développés. Ils se caractérisent par leur multifonctionnalité en répondant à différents types d'enjeux, d'ordre économique et de sécurité alimentaire, récréatif, pédagogique, de santé, d'insertion sociale (Duchemin et al., 2010, Duchemin, 2013). En France, la terminologie relative à ces jardins n'est pas fixée et leurs appellations se recomposent en fonction des époques et des contextes locaux. Une nomenclature relative à une proposition de loi en attente de vote par l'assemblée nationale propose de les classer en jardins familiaux, jardins partagés et jardins d'insertion. Les jardins familiaux sont définis dans cette proposition de loi comme des «terrains divisés en parcelles, lesquelles sont affectées à des particuliers y pratiquant le jardinage pour leurs propres besoins et ceux de leur famille, à l'exclusion de tout usage commercial $»^{1}$, les jardins partagés comme des jardins conçus, cultivés collectivement par les habitants d'un quartier ou d'un village et «ayant pour objet de développer des liens sociaux de proximité par le biais d'activités sociales, culturelles ou éducatives et étant accessibles au public ». Les jardins d'insertion sont eux des outils de réinsertion de personnes en difficulté sociale ou professionnelle. Ils sont reconnus et réglementés par la loi d'orientation du 29 juillet 1998 relative à la lutte contre les exclusions, qui les intègrent parmi les "chantiers d'insertion». Ils s'adressent à des publics dédiés qui ne font pas l'objet de cet article. 
2 Les jardins familiaux apparaissent en France au XIX siècle dans un esprit de catholicisme social et moralisateur (Cabedoce et Pierson, 1996; Dubost, 1997). Qualifiés de jardins ouvriers, industriels ou familiaux selon les acteurs impliqués dans leur création, ils fusionnent en 1952 sous le nom de jardins familiaux. Plébiscités en période de crises comme ce fut, par exemple, le cas pendant les deux guerres mondiales, leur dimension de production alimentaire va perdre de son importance au cours des décennies suivantes, comme c'est le cas également pour les jardins communautaires américains (Monédiare, 1999 ; Pudup, 2008), leurs fonctions se modifient. Ils acquièrent une dimension d'espaces verts et de loisir, mais deviennent aussi la proie de l'urbanisation. En effet, tout comme les terres agricoles, si ces jardins familiaux et partagés ne sont pas protégés, leur existence peut être fragilisée par la pression urbaine. En 1976, leur création et leur protection est favorisée par la possibilité donnée aux collectivités locales et aux Safer d'utiliser leur droit de préemption pour acquérir et aménager ces jardins, et aux associations expropriées d'exiger la mise à disposition d'un terrain équivalent.

3 Les années 2000 sont celles de l'apparition d'une nouvelle forme de jardins collectifs, les jardins partagés. En 1997, le premier forum national du jardinage et de la citoyenneté se tient à Lille, "deux jours d'histoire d'amour avec la terre " ${ }^{2}$. Le rôle des jardins dans les relations sociales, leur lien à la nature, à l'environnement, leur importance dans l'appropriation du cadre de vie y sont fortement affirmés. L'exemple des jardins communautaires de Montréal, de la réappropriation par les citadins des espaces délaissés à New York, y sont développés. Le premier jardin partagé, le jardin des retrouvailles, est créé à Lille (Pathchenko et Consales, 2010) ; la notion de jardin partagé, dont le rôle social est prépondérant, fait ainsi son entrée en France ; ce sont des jardins dont les parcelles sont cultivées collectivement, et qui se fondent sur des valeurs de solidarité, de convivialité, de lien et de partage entre les générations et les cultures ${ }^{3}$, des lieux propices à l'organisation de fêtes et d'ateliers autour du jardinage, autour de thèmes culturels ou artistiques. Ils se multiplient aujourd'hui dans différentes villes européennes, à Paris, Bruxelles, Berlin...

4 Ces jardins collectifs, familiaux et partagés, impliquent différents types d'acteurs mobilisés autour de leur création et de leur gestion, collectivités locales, entreprises publiques ou privées, associations, citadins. L'existence des jardins collectifs se joue donc dans la ville à la croisée des mouvements citoyens et des politiques d'urbanisme et d'environnement. Du fait de l'affirmation de leur rôle social, les municipalités sont tentées de les intégrer dans leur politique d'aménagement, pour proposer à leurs habitants des lieux de récréation où ils peuvent jardiner et nouer des liens sociaux, en particulier de mixité ou intergénérationnels.

Les formes renouvelées des jardins collectifs ont ainsi aujourd'hui de multiples visages, allant du jardin loisir pour le citadin en mal de nature à l'espace d'insertion pour des personnes en difficulté (Bouvier-Daclon, 2001 ; Reyburn, 2006 ; Cérézuelle et Roustang, 2010 ; Duchemin, 2013). La littérature scientifique documente particulièrement leurs fonctions sociales (Dubost, 1997 ; Guyon, 2005), en particulier aux États-Unis (Guitart et al. , 2012), où les concepts de justice alimentaire et sociale sont mobilisés pour aborder la question des jardins dans les quartiers défavorisés (Paddieu, 2012; Shepard, 2013; Ottmann et al., 2012). En France, des travaux sont en cours sur leurs fonctions de production (Pourrias et al., 2013). En 2004, J.-N. Consales avait déjà pointé leur fonction de «laboratoires » pour une agriculture urbaine. Nous nous proposons dans cet article de creuser cette hypothèse sous l'angle des motivations et des pratiques agronomiques des 
jardiniers, et d'analyser en quoi les jardins collectifs urbains constituent des espaces où un renouvellement de la relation entre ville et agriculture serait en germe.

L'agriculture intra-urbaine inclue la culture des jardins collectifs (Duchemin et al. 2010; Grandchamp Florentino, 2012), considérés comme contribuant à l'« agrarisation » de la ville (Salomon Cavin, 2012). Alors que la dépendance des villes vis-à-vis des systèmes de distribution alimentaire globaux s'est accrue au cours des 50 dernières années (Barthel et al., 2013), jardiner en ville contribue à cultiver la ville, à recréer le lien avec la production d'aliments. Mais cette dimension agricole est-elle perçue par les jardiniers lorsqu'ils cultivent leurs jardins, dont la caractéristique première est en effet de permettre au citadin consommateur de participer à l'acte de production alimentaire. Faisant suite à une courte présentation publiée dans Métropolitiques (Scheromm, 2013), cet article a pour objectif d'interroger le jardinage en tant qu'expérience agricole dans la ville d'aujourd'hui. Il s'agit de voir si le jardin fait figure de lieu d'interface entre la ville et l'agriculture, un lieu où s'opèrerait une reconnexion des citadins avec l'acte de produire et de travailler la terre, impliquant peut être un nouveau rapport à la terre et à l'alimentation. Pour ce faire, je m'appuie sur une enquête réalisée auprès de jardiniers membres de jardins collectifs de Montpellier, ville qui a développé une politique active de création de jardins sous la pression de la demande citadine. Le cas de Montpellier est spécifique : les jardins y rencontrent un essor récent, contrairement au cas d'autres villes plus industrielles comme Marseille, où leur histoire est ancienne et où les populations jardinières, de classe populaire, se sont constituées lors de leur établissement (Consales, 2004). Les jardins collectifs montpelliérains se caractérisent par le fait qu'ils constituent des lieux investis par les urbains au cours de la dernière décennie, fait social peu analysé dans la littérature scientifique française. Se pose donc la question de l'identité, des motivations et des pratiques agronomiques de ces nouveaux jardiniers. Ces dernières ont été étudiées au travers d'entretiens semi-directifs dans l'objectif de comprendre quels liens le jardinier urbain montpelliérain établit avec son jardin? Le cultive-t-il principalement dans un objectif de production ou d'autres aspirations sont-elles à l'œuvre? Que cultive-t-il et comment? Quelle vision a-t-il du jardinage et de l'agriculture? Cette vision se répercute-t-elle sur ses pratiques alimentaires, à commencer par ses achats en fruits et légumes?

\section{Matériel et méthodes}

7 Montpellier est la $8^{\mathrm{e}}$ ville française et compte 258000 habitants. C'est une ville sans réelle tradition de jardinage collectif, contrairement au cas des villes du nord de la France où les jardins familiaux sont un héritage du passé industriel (Dubost, 1997). Les jardins familiaux apparaissent en effet peu développés dans l'arc méditerranéen, hormis dans quelques grandes agglomérations industrielles comme Marseille, Gênes ou Barcelone, où leurs fonctions semblaient il y encore quelques dizaine d'années peu reconnues (Consales, 2003). Depuis 2004, les jardins collectifs sont en plein essor à Montpellier : la municipalité a engagé un programme de création de jardins familiaux et partagés géré par son service des espaces verts dans le cadre de sa stratégie de protection de la biodiversité (Scheromm, 2013). Ce dernier a ainsi contribué à la mise en place, au financement et à la gestion de 20 jardins collectifs : trois jardins familiaux représentant un ensemble de 30 à 76 parcelles de $100 \mathrm{~m}^{2}$ environ, soit 158 parcelles au total, et 15 jardins partagés constitués d'une parcelle unique dont la taille varie de 100 à $400 \mathrm{~m}^{2}$, et pouvant, selon les 
jardins, être divisée en mini-parcelles (figure 1). Si les jardins familiaux ont été créés à l'initiative de la municipalité sous la demande des habitants (depuis 2009, la ville a reçu 339 demandes pour les jardins familiaux), la création des jardins partagés est souvent le fruit d'un partenariat entre la Ville et ses "maisons pour tous ", un comité de quartier et un partenaire social, association de sensibilisation à l'environnement ou maison de retraire.

Pour la municipalité, les jardins familiaux sont destinés à l'exercice de la pratique du jardinage pour les familles ne possédant pas de jardin. Les jardiniers, qui doivent habiter à Montpellier et ne pas posséder de jardins, sont sélectionnés par tirage au sort, ce qui induit que le lieu de résidence des jardiniers n'est pas systématiquement associé à celui où se situe le jardin. Vingt parcelles représentant environ $10 \%$ de la totalité des parcelles sont réattribuées en moyenne chaque année pour l'ensemble des trois jardins familiaux gérés par la ville: les jardiniers qui ont la chance d'obtenir un jardin le conservent sur un temps long, et la demande reste donc insatisfaite. Pour les jardins partagés, bien que le roulement soit plus important que dans les jardins familiaux, la demande est également plus importante que l'offre. La municipalité les considère comme des espaces de convivialité où les rencontres intergénérationnelles et la mixité sociale sont favorisées.

9 Les enjeux relatifs à ces jardins collectifs sont donc pour la municipalité d'ordre principalement social et environnemental. Aucun enjeu alimentaire n'est énoncé à leur sujet. Ils sont identifiés comme des outils au service de la protection de la biodiversité et figurent dans le document d'urbanisme qui lui est relatif (Plan de préservation de la biodiversité 2010-2014). Parallèlement à la création des jardins collectifs, la municipalité a dans le cadre de sa stratégie de protection de la biodiversité mis en place une politique d'animation et de sensibilisation dans le domaine de l'environnement. Des animations autour du jardinage biologique (préparation de compost, de purin d'ortie, gestion de l'irrigation...) sont proposées aux jardiniers par des associations mandatées. Concernant les jardins familiaux, la charte signée par les jardiniers stipule que "l'utilisation des produits phytosanitaires doit être conforme à la réglementation en vigueur ». Interrogé sur cette formulation, le service des espaces verts spécifie que l'usage des pesticides est toléré dans la mesure où les applications restent "raisonnées ", et que la charte va évoluer vers la mise en œuvre d'un jardinage exempt de tout pesticide. Pour les jardins partagés, elle préconise de " privilégier une gestion écologique du site, d'éviter les produits phytosanitaires, les pesticides et les engrais chimiques, les gaspillages en eau, de développer le compostage de proximité et la récupération des eaux de pluie, de planter des essences adaptées au sol et au climat ».

Un ensemble de prescriptions techniques, même s'il est à visée environnementale, est ainsi proposé aux jardiniers à l'initiative de la municipalité, impliquée de ce fait dans la définition et le conseil relatif à de bonnes pratiques agricoles. Cette sensibilisation à l'adoption au jardin de pratiques proches de celles de l'agriculture biologique est facteur de construction d'un lien nouveau entre les citadins et l'agriculture.

Quarante entretiens semi-directifs ont été réalisés auprès de jardiniers dans les différents jardins familiaux et partagés de la ville, avec pour objectif de comprendre qui sont ces citadins jardiniers, quelles sont leurs motivations, leurs pratiques agronomiques et leur relation à l'agriculture. Les jardiniers ont été enquêtés au hasard des rencontres lors de passages réalisés sur les jardins, de préférence en fin de journée, le week-end ou pendant les vacances scolaires afin d'avoir l'opportunité de rencontrer des personnes en activité professionnelle. Quelques prises de rendez-vous ont également été réalisées par 
téléphone grâce aux fichiers téléphoniques de jardiniers fournis par des maisons pour tous gestionnaires de jardins partagés : cette technique a été adoptée lorsque, à l'issue de plusieurs passages, aucun jardinier n'avait pu être repéré. Certains jardins partagés présentant des spécificités fortes (quartier défavorisé, jardin cultivé en permaculture), deux personnes maximum y ont été interrogées afin de ne pas donner trop de poids à ces spécificités. L'objectif était d'obtenir un échantillon suffisamment hétérogène pour appréhender les jardiniers dans leur diversité.

12 La diversité des jardiniers a ensuite fait l'objet d'une typologie et a permis de dégager des idéaux-types (Weber, 1951; Soulard, 1999). La démarche idéal-typique de M. Weber, développée dans une approche comparative explicative des phénomènes sociaux, s'appuie sur l'observation du phénomène étudié, ici la nature de la forme d'engagement du jardinier dans l'activité de jardinage, pour dégager des profils théoriques en permettant l'analyse.

Figure 1. Exemples de jardins

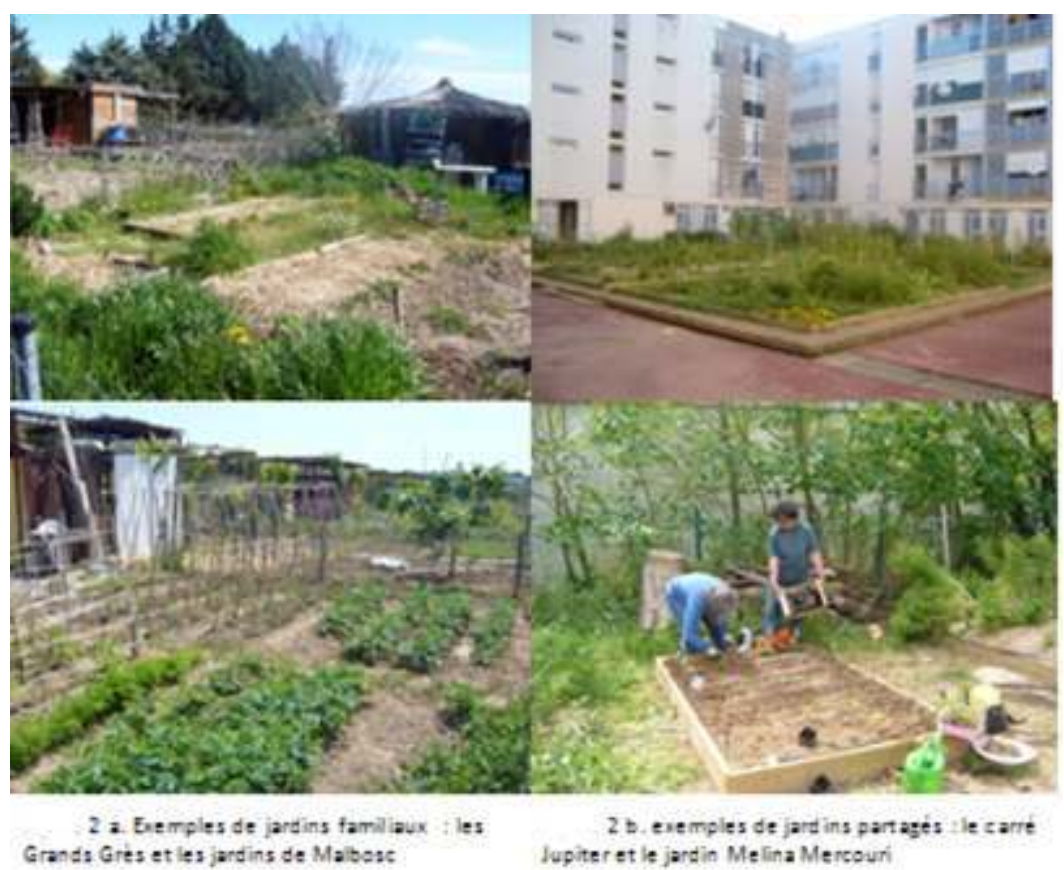

DANS LeS JARDINS FAMILIAUX (2A), LES PARCELLES LOUÉES SE PRÉSENTENT SOUS LA FORME d'UN CARRÉ CLÔTURÉ ET SONT LIVRÉES AVEC CABANON, PERgOLA ET RÉCUPÉRATEUR D'EAU de PLUIE. LES JARDINS PARTAgÉS (2B) PRÉSENTENT DES FORMES PLUS DIVERSIfIÉES, gRANDE PARCELLE CULTIVÉE COLLECTIVEMENT OU SUBdiVISÉE EN PLUSIEURS MINI-PARCELLES ATTRIBUÉES CHACUNE À deS JARDINIERS.

\section{Des jardiniers aux profils diversifiés}

Les jardiniers interrogés dans les jardins familiaux et partagés se différencient par leur âge, leur genre et leurs catégories socio-professionnelles (tableau 1). Les hommes sont très largement majoritaires dans les jardins familiaux (17/20) où la moyenne d'âge des jardiniers est de 57 ans. Ils sont par contre minoritaires (5/20) dans les jardins partagés où leur moyenne d'âge est également supérieure à la cinquantaine, soit 51 ans. Les jardiniers des jardins familiaux sont pour les $2 / 3$ retraités, ouvriers ou employés. Six d'entre eux sont de catégorie socio-professionnelle moyenne ou supérieure. Tous, à 
l'exception d'une jardinière, ont jardiné dans leur enfance, confirmant le fait que le jardinage est souvent un héritage culturel (Guyon, 2008). Les jardiniers des jardins partagés comptent environ $1 / 3$ de retraités et de personnes sans emploi pour $2 / 3$ d'actifs appartenant principalement aux catégories socio-professionnelles intermédiaires. Il s'agit pour moitié d'entre eux de leur première expérience de jardinage.

La très large majorité des jardins est de nature potagère, que ce soit dans les jardins familiaux ou dans les jardins partagés. Certains jardiniers donnent cependant à leur jardin une dimension ornementale importante, cultivant des fleurs, plantant des arbres ou des buissons ornementaux

Tableau 1. Catégories socio-professionnelles des jardiniers enquêtés

\begin{tabular}{|l|l|l|}
\hline $\begin{array}{l}\text { Catégories socio- } \\
\text { professionnelles }\end{array}$ & $\begin{array}{l}\text { Jardins } \\
\text { familiaux }\end{array}$ & Jardins partagés \\
\hline Ouvriers et employés & $4 / 20$ & $3 / 20$ \\
Professions intermédiaires & $5 / 20$ & $6 / 20$ \\
Professions supérieures & $1 / 20$ & $3 / 20$ \\
Sans emploi & - & $2 / 20$ \\
Retraités & $10 / 20$ & $6 / 20$ \\
\hline
\end{tabular}

15 Les retraités constituent donc un groupe très présent parmi les jardiniers, en particulier dans les jardins familiaux, alors qu'ils représentent $4,7 \%$ de la population de la ville (Insée, 2011). Le jardinage reste donc une activité de loisir très prisée par ces derniers (cf. Guyon, 2008 et Duchemin, 2013). Peu de personnes sans emploi semblent par contre pratiquer le jardinage, alors que leur population est d'environ $10 \%$ à Montpellier. On en trouve cependant deux dans les jardins partagés qui sont d'accès gratuit, contrairement aux jardins familiaux pour lesquels les jardiniers paient une location. Les actifs, qui représentent dans l'échantillon environ la moitié des jardiniers interrogés, appartiennent principalement aux catégories intermédiaires, ce qui témoigne de l'intérêt que représente pour ces derniers le jardinage urbain, en particulier en jardin partagé.

\subsection{Les motivations des jardiniers}

Les jardins collectifs, reflet de l'engouement récent des citadins pour le jardinage, offrent un terrain d'observation et d'étude de leurs motivations. Ces dernières sont présentées dans le tableau 1.

17 L'envie de faire, de voir pousser ses propres légumes - des légumes qui ont du goût alliées aux notions de plaisir, de passion et de bonheur, sont les éléments les plus mis en avant par les jardiniers. Elles s'associent aux besoins exprimés d'être dehors, dans la nature, au besoin de rapport physique et sensible avec la terre et le végétal (exprimé surtout dans les jardins familiaux) :

"C'est une vraie passion, j'ai beaucoup de plaisir à produire, à être dehors, le végétal j'adore, les couleurs, les transparences " (jardinière en jardin familial, 38 ans).

Puis viennent les notions de passer, d'occuper le temps, de ressourcement, de recherche de sérénité dans un espace de nature dont la conception peut répondre à des critères esthétiques, suggérant que le temps passé au jardin est fondamental pour le bien-être du 
jardinier :

"Je préfere être ici que de regarder la télé, aller au café c'est pas mon genre". "Je viens ici pour passer le temps, avec mes enfants pour la grillade, samedi et dimanche, je reste tranquille au jardin » (jardinier en jardin familial, 50 ans).

Tableau 2. Caractéristiques des motivations des jardiniers dans les jardins familiaux et partagés de Montpellier

\begin{tabular}{|l|l|l|}
\hline Nature de la motivation & $\begin{array}{l}\text { Jardins familiaux } \\
\text { Nombre de jardiniers /20 }\end{array}$ & $\begin{array}{l}\text { Jardins partagés } \\
\text { Nombre de jardiniers/20 }\end{array}$ \\
\hline Motivations pour le jardinage & 11 & 7 \\
Faire pousser soi-même des légumes & 13 & 7 \\
Plaisir, passion, bonheur & 10 & 13 \\
Besoin de nature/être dehors & 11 & 5 \\
Rapport physique à la terre ou au végétal & 7 & 4 \\
Passe-temps & 6 & 4 \\
Lieu de ressourcement, de sérénité & 4 & 5 \\
Faire que la parcelle soit jolie & 2 & 10 \\
Lieu de rencontre & 1 & 1 \\
Activité physique & & 4 \\
Transmettre des pratiques aux plus jeunes & & \\
Motivations plus générales dans & & 5 \\
lesquelles le jardinage prend sens & 5 & 5 \\
Construire une autre société & 8 & \\
Préoccupations environnementales globales & & \\
\hline
\end{tabular}

Si ces motivations sont présentes à la fois dans les jardins familiaux et partagés, la recherche de lien social, très peu citée dans les jardins familiaux, apparaît importante dans les jardins partagés, en rapport avec l'envie de partager la passion du jardinage et les connaissances acquises avec les plus jeunes. Cet argument, souvent développé par des femmes jardinières, pourrait être une raison explicative de leur penchant pour les jardins partagés. De plus, les parcelles de petite taille proposées dans ces derniers semblent correspondre davantage aux aspirations féminines que les parcelles des jardins familiaux demandant davantage de travail et d'investissement.

Les motivations liées à la pratique du jardinage s'inscrivent parfois dans des motivations d'ordre plus large. Celles-ci peuvent être relatives à l'existence d'une conscience environnementale globale, donnant lieu à des comportements éco-responsables où la pratique du jardinage trouve un sens :

"Je fais attention à ce que je mange par rapport à l'environnement, je trie mes déchets, je respecte la nature et l'environnement dans ma vie quotidienne ", "J'ai l'impression de participer à quelque chose de planétaire quand je jardine ». (jardinier en jardin partagé, 45 ans).

Elles peuvent également s'insérer dans un projet de construction d'un nouvel ordre social. Le jardinage est alors vu comme pouvant participer à l'élaboration d'une société plus durable et solidaire. S'exprime alors une conception vivrière du lien que le jardinier établit avec son jardin :

"On est au bout du système, je me prépare une autre vie où on est plus autonome et le jardinage en fait partie"; " On réfléchit à comment vivre en autarcie, nous on a pas envie d'être des brebis et on est complétement dépendant de l'industrie"; "C'est une réappropriation de ce que l'on mange, c'est un acte militant " (jardinier en jardin familial, 60 ans). Les motivations des jardiniers s'avèrent donc variées, et rejoignent pour partie celles mises en avant dans les jardins communautaires de Montréal (Duchemin, 2013). Le jardinage est considéré par les jardiniers comme un simple passe-temps, un loisir, un 
plaisir ressourçant associé à l'immersion dans une nature agricole produisant des légumes de qualité, à savoir ayant du goût, ou encore comme un engagement d'ordre politique dans la construction d'une société plus éco-responsable, moins industrielle, où l'agriculture vivrière et familiale retrouve une place pour le bénéfice de chacun. Cette dernière argumentation est donnée par des jardiniers appartenant aux catégories socioprofessionnelles intermédiaires et supérieures. À noter qu'aucun des jardiniers interrogés ne dit jardiner pour satisfaire des besoins alimentaires, certains précisant même que le jardinage représente un véritable coût.

22 La recherche de convivialité se dégage comme une caractéristique des jardins partagés. Cette constatation se doit cependant d'être modulée. Des travaux sur les relations se nouant entre jardiniers dans les jardins collectifs montrent en effet que ces dernières restent souvent superficielles et que les jardins collectifs ne constituent que des « espaces de sociabilité réduite » (Bouvier-Daclon et Sénécal, 2001 ; Kurtz, 2001).

\subsection{Les pratiques agronomiques des jardiniers}

23 Si les jardiniers se différencient par leur statut social, par leurs motivations, ils diffèrent également par leurs pratiques agronomiques (tableau 2).

24 Concernant le travail du sol, les jardiniers œuvrent en général manuellement, à la bêche et à la pioche. Mais certains choisissent de laisser leur parcelle en herbe et de toucher le moins possible au sol:

"Je ne bine pas, je ne retourne pas la terre, j'apporte en surface les herbes et les déchets de jardinage, je laisse faire; je pense que conserver un équilibre faune et flore est très important. » (jardinière en jardin partagé, 36 ans).

25 Pour l'amendement du sol, aucun jardinier n'utilise d'engrais de synthèse. Ils fabriquent presque tous leur propre compost et utilisent également du fumier. Certains jardiniers se démarquent en nommant leurs pratiques, et se positionnent comme pratiquant la permaculture ou le jardinage biologique.

26 Les jardiniers ne parlent jamais spontanément des maladies des plantes au jardin. De manière générale, la pression parasitaire y semble assez faible et n'est pas un sujet de préoccupation fort, du fait que l'objectif de production n'est pas prédominant. Cependant, ils se différencient dans leur manière de soigner les plantes. Si l'utilisation de pesticides de synthèse autres que la bouillie bordelaise ou le sulfate reste marginale, ces derniers sont utilisés par environ $1 / 3$ des jardiniers des jardins familiaux. La moitié certifie n'employer aucun produit phytosanitaire, arrache les plantes malades, écrase les pucerons et retire les escargots à la main. L'utilisation de plantes comme les œillets d'Inde ou les capucines sont parfois mises en avant pour leur intérêt phytosanitaire. Certains jardiniers des jardins partagés préparent également des tisanes, du purin d'ortie ou de prêle afin de soigner les plantes malades.

27 Concernant l'arrosage, l'eau de pluie est récupérée par la très grande majorité des jardiniers, qui sont tous dans une démarche de maitrise de l'utilisation de l'eau, certains installant même des systèmes de goutte à goutte ou pratiquant le paillage.

Ils accordent peu d'attention aux choix des variétés, excepté pour les tomates, dont les variétés " cœur de bœuf », "vertes », « noires de Crimée », " tomates cerise », " poire » sont très appréciées pour la diversité des goûts et des formes qu'elles proposent :

"Les variétés, on les choisit comme ça, en fonction de ce qu'on nous conseille au magasin " 


\subsection{Liens entre jardinage, agriculture et type d'achat alimentaire}

33 À la question «quels liens voyez-vous entre jardinage et agriculture ? ", 17 jardiniers, 10 de jardins familiaux et 7 en jardins partagés répondent qu'ils envisagent leur pratique du jardinage comme une pratique pouvant être identifiée à une pratique agricole :

"Oui, ce que je fais ici, c'est de l'agriculture, mais je ne fréquente pas d'agriculteurs, ils ne font pas partie de mon réseau social » (jardinier en jardin partagé, 52 ans).

"Oui, c'est de l'agriculture, on doit réapprendre, on a été dépossédé et c'est indispensable à la vie, c'est important de maintenir une production de nourriture dans la ville ». (jardinier en jardin familial, 38 ans).

Les autres ne voient aucun lien entre agriculture et jardinage. De leur point de vue, la différence n'est pas associée à l'échelle de production, mais davantage aux fonctions du jardinage, vues comme éducationnelle, récréative, environnementale :

«Ici, ce n'est pas de l'agriculture, c'est écologique et éducationnel » (jardinière en jardin partagée, 58 ans).

«ici, j'ai pas l'impression de faire de l'agriculture, c'est juste un plaisir » (jardinier en jardin familial, 61 ans).

35 À la question « que pensez-vous de l'agriculture ? ", les jardiniers répondent de manière homogène. L'agriculture est perçue une activité intensive, polluante. Il existe dans le regard de tous les jardiniers la «bonne agriculture» (l'agriculture familiale, celle d'autrefois) par opposition à la «mauvaise agriculture» (l'agriculture intensive, celle qu'il faudrait changer car " on ne sait plus ce que l'on mange ») :

"J'associe l'agriculture à une image polluante. Cela représente les excès de notre société, avec une surexploitation de la terre. J'espère ici faire la nouvelle agriculture, celle vers laquelle on devrait tendre » (jardinier en jardin partagé, 60 ans).

36 Dans leurs pratiques de jardinage, ils s'inscrivent dans un lien d'opposition avec cette agriculture industrielle, soulignant que leurs pratiques sont respectueuses de l'environnement, davantage centrées sur l'obtention d'un aliment de qualité que sur la productivité, exprimant là encore que leur préoccupation n'est pas de se nourrir grâce aux produits du jardinage.

37 À la question «Accordez-vous de l'importance à la provenance des légumes que vous achetez?", les réponses des jardiniers se répartissent en trois catégories. La première catégorie correspond aux jardiniers achetant quasi exclusivement des légumes à la fois de proximité et issus de l'agriculture biologique ( 5 pour les jardins familiaux; 5 pour les jardins partagés). Ce sont en particulier les jardiniers investis dans les pratiques les plus alternatives. La deuxième catégorie correspond aux jardiniers présentant un comportement mixte, achetant des produits issus de l'agriculture biologique de manière non-régulière ( 4 pour les jardins familiaux; 6 pour les jardins partagés). Ces deux premières catégories comprennent principalement des jardiniers appartenant aux catégories socio-professionnelles intermédiaires et supérieures. Enfin, la troisième catégorie correspond aux jardiniers achetant des produits issus de l'agriculture conventionnelle, principalement en supermarchés (11 pour les jardins familiaux, 9 pour les jardins partagés). Ils justifient leur choix soit par le fait que «le bio, ça coûte trop cher, ce n'est pas pour tout le monde", soit par le fait qu'ils ne croient pas que ces produits soient réellement « bio ", soit parce qu'ils ne font pas vraiment de rapport entre la manière dont ils cultivent et ce qu'ils achètent. 
ont donc différentes conceptions du jardinage et de leur lien avec l'agriculture qui s'expriment au travers des motivations, des pratiques et des points de vue recueillis. Au sein de cette diversité se détache cependant l'image d'un jardinier urbain : impliqué dans des pratiques respectueuses de l'environnement sur la parcelle qu'il cultive, même si elles ne lui sont pas imposées comme cela est le cas pour les jardins familiaux, pour lesquels la charte mise en place par la Ville autorise les produits phytosanitaires dans un usage raisonné, exprimant un besoin essentiel de nature nécessaire à son équilibre, qui expérimente, mobilise de nouvelles connaissances pour s'engager dans un travail de production de type agriculture biologique.

\section{Vers une typologie relevant de différentes formes d'engagement dans la pratique du jardinage}

39 L'identification de profils théoriques de jardiniers permet d'approfondir la nature de la relation existant entre les jardiniers, leurs pratiques et le lien qu'ils établissent à l'agriculture.

40 La diversité des jardiniers montpelliérains jardinant dans les jardins collectifs a ainsi appréhendée au travers de la typologie de leurs discours. De l'analyse des variables explorées: motivations, pratiques agronomiques, vision de l'agriculture et type d'achat en fruits et légumes, se dégagent trois profils théoriques ou types-idéaux. Ces profils théoriques correspondent à trois formes d'engagement dans l'activité de jardinage, et se différencient par les quatre variables citées ci-dessus et les liens qu'on peut établir entre elles (tableau 3).

\subsection{Idéal type du jardinier du dimanche}

41 Cet idéal-type se rencontre principalement dans les jardins familiaux. Ce jardinier de sexe masculin, retraité ouvrier ou de classe moyenne, vient passer du bon temps au jardin tout en cultivant ses légumes. Il voit le jardinage comme un passe-temps. Il associe son jardin à un bout de campagne où il fait bon prendre l'air, s'asseoir sur une chaise, discuter avec ses voisins ou passer du temps en famille. L'espace du jardin peut ainsi devenir celui d'une nature ludique et ordonnée, s'apparentant à un espace de loisirs avec cabanon aménagé, parfois transformé en pagode, terrasse bétonnée (interdite au règlement...), barbecue, table et chaise. On y prend l'apéro, on y mange, on y organise des festivités. Il est extension de la sphère domestique. Ce jardinier bêche son jardin, retournant la terre chaque année comme il l'a vu faire par ses parents, arrose et entretient son morceau de pelouse, traite ses tomates à la bouillie bordelaise, sulfate sa vigne grimpante, à la fois décorative et productive. Sa récolte est parfois conséquente, il congèle alors ses légumes, ce qui lui permet de tendre vers une autonomie. Pour compléter cette récolte qu'il considère comme produite naturellement, il achète des légumes au supermarché de proximité, sans prêter particulièrement d'attention à leur provenance ni aux techniques de production.

Récit idéal-typique d'un jardinier du dimanche 
Louis, 65 ans, jardinait déjà quand il était petit. Il est routier retraité, vit en appartement et a obtenu sa parcelle dans un jardin familial depuis qu'il est retraité. Il l'a aménagé en terrain de loisir où il vient faire des grillades en famille, « avec deux bassins, un magnolia et deux oliviers, une treille pour le raisin, des fleurs pour décorer ». Il a également mis en place un carré avec des tomates, des courgettes, des aubergines, des radis et des piments. Il retourne chaque fin d'hiver la terre à la bêche et $\mathrm{y}$ apporte du fumier qu'il ramasse dans un champ où paît un cheval dans un village proche de Montpellier. Il achète très peu de semences et utilise les graines qu'il récupère dans les légumes qu'il produit. Ici, il est dehors, il passe le temps, il parle avec ses voisins jardiniers. Le jardin est pour lui une occupation, un espace de loisir, de bricolage plus qu'un espace de production, bien qu'il ait plaisir à déguster ce qu'il produit. Pour avoir de belles plantes, il traite quand cela est nécessaire, quelquefois avec des pesticides de synthèse. Pour compléter sa production, il achète des légumes au supermarché, ce qu'il trouve. Il ne voit pas vraiment l'intérêt d'acheter des légumes issus de l'agriculture biologique, il ne croit d'ailleurs pas que ces légumes soient vraiment produits selon le cahier des charges exigé pour la certification. « On ne peut jamais savoir d'où ça vient, tout est bidon ». Il pense que l'agriculture industrielle produit des aliments de mauvaise qualité, mais que pour que tout le monde puisse manger à sa faim, il faut en passer par là.

\subsection{Idéal-type du jardinier hédoniste}

Le jardinier hédoniste est un citadin attaché à la vie urbaine, en mal de nature. Il trouve dans la pratique du jardinage un moyen d'expression et de ressourcement mobilisant la terre et le végétal, lui permettant de lutter contre les tensions générées par la vie urbaine. La parcelle de petit format lui convient car elle est adaptée au temps qu'il peut lui consacrer. Il jardine donc plutôt en jardin partagé, mettant en œuvre des techniques respectueuses de l'environnement car sensibilisé aux pratiques de l'agriculture biologique. Le jardin est pour lui un espace approprié collectivement où les relations entre jardiniers sont source de motivation. Le plaisir pris au jardin à s'occuper des plantes n'est pas associé à un objectif de production. Ce jardinier, plutôt de sexe féminin et de catégorie socio-professionnelle intermédiaire, produit souvent assez peu et sa consommation de légumes du jardin est anecdotique. Il aime partager la récolte et échanger conseils et recettes de jardinage. Il achète parfois des légumes locaux ou issus de l'agriculture biologique, mais n'en fait pas une habitude. Il s'agit souvent de sa première expérience de jardinage et cette découverte est source de joie et de plaisir. L'espace du jardin constitue pour lui un espace de nature agricole et de convivialité.

Récit idéal-typique d'un jardinier hédoniste 
Éva, 35 ans, est infirmière. Célibataire, elle habite un petit appartement dans la vieille ville. Elle peut venir à pied au jardin partagé de proximité. Elle n'avait pas de connaissance du jardinage quand elle s'est inscrite au jardin. Elle prend beaucoup de plaisir à jardiner, regarder germer les graines et voir pousser les plantes, à apprendre les pratiques agronomiques de l'agriculture biologique au cours des ateliers mis en place par l'association mandatée par la ville. Dans la petite parcelle qui lui a été attribuée, elle travaille à la grelinette pour ne pas trop remuer la terre, composte ses déchets ménagers pour améliorer le sol, achète des semences et des plants biologiques ou récupère ses semences dans les légumes qu'elle achète ou cultive. Elle ne traite pas du tout ses cultures et préfère perdre des plantes : son objectif n'est pas de récolter, mais de faire pousser, pour ensuite " partager avec les autres jardiniers, faire profiter les enfants des fraises ». Pour protéger ses cultures, elle plante des œillets d'Inde, qui repoussent les insectes et protègent ses cultures. Elle aime venir au jardin le jour où les enfants des écoles sont présents, afin de transmettre ce qu'elle a appris. Elle «prend plaisir à voir pousser les plantes, à rendre un coin de la ville plus joli ». Elle achète parfois des légumes issues de l'agriculture biologique, mais leur prix est trop élevé pour qu'elle en fasse une habitude. Elle a la sensation, «oui pourquoi pas, de faire une forme d'agriculture réfléchie, biologique, allant dans le bon sens, même si cela tient plutôt de l'éducationnel que de l'agricole. »

\subsection{Idéal-type du jardinier militant}

Qu'il jardine en jardin familial ou partagé, la pratique du jardinage est pour le jardinier militant une mise en application de valeurs et d'idées. Il inscrit sa pratique dans une perspective de changement de société, en particulier concernant les modes de production et de consommation alimentaire. Ce jardinier alternatif est convaincu que l'économie de marché ne répond pas aux aspirations humaines et prépare un changement social qu'il appelle de ses vœux, et qui parfois participe d'une logique libertaire. Il présente une conscience environnementale très affirmée, se démarque par un comportement d'achat de produits locaux issus de l'agriculture biologique sur les marchés ou dans les magasins bio. Il utilise des techniques culturales proches de la permaculture ou de l'agro-écologie, minimise le travail du sol pour respecter son équilibre faune-flore. Il est de catégorie socioprofessionnelle intermédiaire ou supérieure.

Récit idéal-typique d'un jardinier militant 
Paul a 45 ans, il est graphiste et sculpteur, sa famille recomposée compte 3 enfants. Il habite en cœur de ville et vient d'obtenir une parcelle de jardin familial qu'il demande depuis 7 ans auprès de la mairie. Le fait de jardiner lui rappelle son enfance. Il aime la nature, travailler la terre, être au jardin en famille; ses enfants peuvent y profiter d'un espace de nature et de production. Il achète ses semences biologiques en jardinerie ou les fabriquent. Dans le jardin, les semences s'échangent entre un petit groupe de jardiniers. Pas vraiment adepte du fumier qu'il considère comme trop riche en azote, il utilise du compost qu'il fabrique pour nourrir son sol et précise qu'il n'utilise aucun engrais chimique. Il retourne la terre le moins possible, paille pour minimiser l'arrosage et conserver la terre humide. Le jardin est pour lui un espace où il improvise, expérimente, teste de nouvelles manières de faire. Son jardin a été surnommé «la jungle, il y a de tout partout, il n'est pas ordonné mais il est pensé, il y a des associations de plantes, de la phacélie pour les abeilles ». Il achète et mange des légumes issus de l'agriculture biologique, de préférence à des producteurs qu'il connaît. Il pense que le système en place n'est plus durable, que tout va changer d'ici une vingtaine d'années; cultiver son jardin est pour lui une manière de préparer ce changement, en s'inscrivant dans une stratégie collective de réappropriation de la production alimentaire.

Tableau 4. Caractéristiques des profils théoriques de jardiniers. Correspondances avec les quatre variables explorées : motivations, pratiques agronomiques, vision de l'agriculture et type d'achat en fruits et légumes

\begin{tabular}{|c|c|c|c|c|}
\hline $\begin{array}{l}\text { Types-idéaux } \\
\text { (catégorie socio- } \\
\text { professionnelle) }\end{array}$ & Motivations & $\begin{array}{l}\text { Pratiques } \\
\text { agronomiques }\end{array}$ & $\begin{array}{l}\text { Vision } \\
\text { de l'agriculture }\end{array}$ & $\begin{array}{l}\text { Fruits et } \\
\text { légumes } \\
\text { achetés }\end{array}$ \\
\hline $\begin{array}{l}\text { Jardinier du } \\
\text { dimanche } \\
\text { (retraités, } \\
\text { ouvriers, } \\
\text { employés, } \\
\text { professions } \\
\text { intermédiaires) }\end{array}$ & $\begin{array}{l}\text { Passe-temps } \\
\text { Prendre l'air } \\
\text { Se réunir en } \\
\text { famille ou entre } \\
\text { amis } \\
\text { Lien social }\end{array}$ & $\begin{array}{l}\text { Bêchage } \\
\text { Traitement contre les } \\
\text { maladies avec parfois } \\
\text { utilisation de } \\
\text { pesticides }\end{array}$ & $\begin{array}{l}\text { Critique modérée } \\
\text { de l'agriculture } \\
\text { industrielle et } \\
\text { consommation de } \\
\text { produits issus de } \\
\text { cette dernière }\end{array}$ & $\begin{array}{l}\text { Issus de } \\
\text { l'agriculture } \\
\text { industrielle } \\
\text { (achat en } \\
\text { supermarché) }\end{array}$ \\
\hline $\begin{array}{l}\text { Jardinier } \\
\text { hédoniste } \\
\text { (retraités, } \\
\text { professions } \\
\text { intermédiaires,) }\end{array}$ & $\begin{array}{l}\text { Plaisir de produire } \\
\text { Contact avec la } \\
\text { nature } \\
\text { Ressourcement } \\
\text { Lien social }\end{array}$ & $\begin{array}{l}\text { Bêchage / Retourner } \\
\text { le sol le moins } \\
\text { possible } \\
\text { Souci du sol et de } \\
\text { respect de l'équilibre } \\
\text { faune/flore } \\
\text { Pas de traitement ou } \\
\text { traitements à base de } \\
\text { produits naturels } \\
\text { Association de } \\
\text { plantes }\end{array}$ & $\begin{array}{l}\text { Critique de } \\
\text { l'agriculture } \\
\text { industrielle avec } \\
\text { efforts d'achat de } \\
\text { produis de } \\
\text { proximité et issus } \\
\text { de l'agriculture } \\
\text { biologique }\end{array}$ & $\begin{array}{l}\text { Issus de } \\
\text { l'agriculture } \\
\text { industrielle } \\
\text { (achat en } \\
\text { supermarché) } \\
\text { Issus de } \\
\text { l'agriculture } \\
\text { biologique } \\
\text { Produits } \\
\text { locaux }\end{array}$ \\
\hline $\begin{array}{l}\text { Jardinier } \\
\text { militant } \\
\text { (professions } \\
\text { intermédiaires et } \\
\text { supérieures) }\end{array}$ & $\begin{array}{l}\text { Passion/plaisir de } \\
\text { produire } \\
\text { Contact avec la } \\
\text { nature } \\
\text { Politiques: } \\
\text { réappropriation de } \\
\text { son alimentation, } \\
\text { protection de } \\
\text { l'environnement, } \\
\text { changement } \\
\text { social }\end{array}$ & $\begin{array}{l}\text { Retourner le sol au } \\
\text { minimum. } \\
\text { Souci du sol et de } \\
\text { respect de l'équilibre } \\
\text { faune/flore } \\
\text { Pas de traitement ou } \\
\text { traitements à base de } \\
\text { produits naturels } \\
\text { Association de } \\
\text { plantes }\end{array}$ & $\begin{array}{l}\text { Critique forte de } \\
\text { l'agriculture } \\
\text { industrielle et rejet } \\
\text { des légumes issus } \\
\text { de cette dernière }\end{array}$ & $\begin{array}{l}\text { Issus de } \\
\text { l'agriculture } \\
\text { biologique, } \\
\text { achetés à des } \\
\text { producteurs } \\
\text { locaux } \\
\text { identifiés }\end{array}$ \\
\hline
\end{tabular}

Les trois figures de jardiniers se dégageant de l'analyse de leur engagement dans l'activité se différencient ainsi par leurs motivations, en lien avec leurs pratiques de jardinage, leur vision de l'agriculture et leurs comportements d'achat en légumes, issus ou non de l'agriculture biologique. Les jardiniers correspondant au premier idéal-type jardinent principalement pour passer le temps; les $2^{\mathrm{e}}$ trouvent dans la pratique du jardinage un moyen d'expression leur permettant d'approcher la nature en contexte urbain; pour les 
troisièmes, le fait de jardiner relève d'un véritable engagement dans une pratique agricole, ou plus largement dans la construction d'une société où l'agriculture familiale retrouverait une place de choix. Aucun de ces jardiniers ne jardine dans un objectif de sécurité alimentaire ; on retrouve dans leurs motivations de fortes similitudes avec celles observées de manière détaillée par Reyburn (2006) dans les jardins communautaires de Montréal, à la différence cependant que les jardiniers montpelliérains semblent peu concernés par la fonction verdissement de leur quartier.

\section{Conclusion}

Si le jardin propre et ordonné où le jardinier affirme son estime de soi (Weber, 1998) reste un modèle encore observable - celui du jardinier du dimanche - la typologie des jardins et de leurs jardiniers évolue avec le temps, et s'ajuste au contexte social et technique de son époque. Les jardiniers considèrent toujours pour partie leurs jardins comme une extension de la sphère domestique, mais aussi comme des lieux d'apprentissage et d'immersion dans un environnement naturel qu'il s'agit de mieux connaître et maitriser, et pour certains comme des espaces d'engagement politique ou de reprise en main de leur alimentation. La typologie de jardiniers montréalais réalisé par Reyburn (2006) met en exergue une catégorie voisine de celle du jardinier militant, une catégorie où les jardiniers sont "conscients des bonnes habitudes alimentaires, des comportements respectueux de l'environnement ", mais où la dimension militante est cependant moins apparente. Cette différence peut être expliquée par la dizaine d'années séparant les résultats, la différence culturelle entre les deux pays, et le fait que les classes socioprofessionnelles intermédiaires et supérieures sont bien présentes dans l'échantillonnage montpelliérain, démontrant que de nouvelles catégories sociales s'intéressent au jardinage, alors que cette activité concernait il y a encore quelques années majoritairement les classes ouvrières ou les retraités (Consales, 2003; Guyon, 2005). Le jardin, symbole de son temps et trait d'urbanité (Quellier, 2012), se façonne ainsi à l'image du jardinier qui le cultive. Forme d'expression de nouveaux mouvements sociaux, il matérialise des tendances sociales sous-jacentes jusqu'à devenir parfois une " expérience artistique contre-culturelle », une " zone d'autonomie temporaire ou de contre-pouvoir » (Pereira, 2009).

À la différence des typologies évoquées ci-dessus, la typologie présentée dans cet article est centrée sur les pratiques agronomiques des jardiniers, au sein desquelles les principales différences correspondent à la manière dont ils travaillent le sol et dont ils soignent les plantes. Ces indicateurs révèlent différentes formes d'engagement dans la pratique du jardinage : le bêchage et l'utilisation possible de pesticides caractérisent le jardinier du dimanche, le non-travail du sol et les traitements à base de produits naturels les jardiniers hédonistes et militants, avec une attention plus marquée pour le respect du sol concernant le jardinier militant. Dans mon échantillon, le profil socio-culturel et l'âge sont des déterminants forts de la forme d'engagement dans la pratique du jardinage. Les jardiniers retraités ou ouvriers pratiquent plutôt un jardinage de loisir traditionnel. Les plus jeunes, appartenant à des catégories socio-professionnelles moyennes et supérieures, développent des pratiques plus alternatives; ils recherchent de nouvelles manières de cultiver faisant appel au fonctionnement des écosystèmes biologiques. Ils expérimentent, testent, pour mieux valoriser les phénomènes biologiques qu'ils observent et mettent en évidence. Ces jardiniers peuvent nommer leur pratique, s'inscrivant dans des « courants » 
agronomiques comme ceux de l'agriculture biologique, de l'agro-écologie ou de la permaculture, dont ils maîtrisent plus ou moins bien les concepts. Dans leur ensemble, les jardiniers disent attacher une grande importance à produire des aliments sains, sans utilisation de produits de synthèse. Leur préoccupation commune est de pratiquer un jardinage exempt ou très économe en produits phytosanitaires. Pour autant, sortis du jardin, ils s'insèrent dans le modèle de consommation dominant, pour des raisons financières ou de commodité, hormis dans le cas du jardinier militant, qui lui dit ne consommer que des produits locaux et issus de l'agriculture biologique.

Ces différents portraits sont ainsi révélateurs de la relation du citadin jardinier à son jardin, en démontre la multifonctionnalité et l'intérêt qu'il suscite auprès de différents publics citadins : hommes ou femmes, retraités ou appartenant à différentes classes socioprofessionnelles, inférieures, mais aussi intermédiaires ou supérieures. Ces portraits traduisent également l'appréhension qu'a le jardinier de l'environnement urbain, qu'il contribue à redessiner au travers de ses activités.

Aux États-Unis, où la production alimentaire à grande échelle (community food production) est très documentée (McClintock, 2010 ; Corrigan M.-P., 2011 ; Guitard et al., 2012 ; Smith et al., 2013 ; Vitiello and Wolf-Powers, 2014), des individus ou des communautés toujours plus nombreux s'engagent dans la production de nourriture dans un objectif de sécurité alimentaire. Si l'essor des jardins collectifs à Montpellier constitue bien une illustration de celui de cette production à grande échelle, l'enjeu de sécurité alimentaire n'y est cependant pas prioritaire, comme nous l'avons vu plus haut. Sa principale fonction est une fonction de nature, aujourd'hui élément essentiel au bien-être du citadin (BourdeauLepage et Vidal, 2013). Or, dans nos sociétés urbaines individualistes, la nourriture est sans doute ce qui nous relie le plus à la nature (Francis et al., 2003). Les propos recueillis auprès des jardiniers soulignent que les jardins collectifs sont un élément de cette nature urbaine, dont la particularité est d'être potagère. En cultivant des plantes alimentaires, les jardiniers se rapprochent de la nature, participent au verdissement et à la renaturalisation de la ville, à l'équilibre de son métabolisme et à l'amélioration du cadre de vie des citadins (Reyburn, 2002). Mais si les jardins collectifs représentent pour les citadins montpelliérains interrogés des lieux de plaisir et de bien-être, de contact avec la terre, les plantes et la nature, les résultats obtenus montrent qu'ils sont également vécus comme des lieux d'engagement dans une pratique agricole. Bien que le lien établi avec l'espace du jardin ne soit pas prioritairement alimentaire, il est en effet qualifié d'agricole par environ la moitié des jardiniers de l'échantillon. Une connexion s'y construit donc entre le citadin jardinier et l'agriculture au travers de l'acte de production, de la volonté de maîtrise des ressources naturelles, en mobilisant des pratiques (travail et amélioration $\mathrm{du}$ sol, ensemencement et plantation, arrosage...), en entrant dans un processus d'apprentissage et d'expérimentation. De nouvelles pratiques respectueuses de l'environnement y sont mises en œuvre. La municipalité contribue à renforcer cette connexion en proposant des animations de jardinage où sont enseignés les préceptes de l'agriculture biologique, de l'agro-écologie ou de la permaculture.

Les jardins collectifs représentent donc des espaces d'interface entre ville, nature et agriculture permettant au citadin de se reconnecter avec celle-ci, ses pratiques et ses réalités. Le jardinage s'avère ainsi participer d'un mode d'habiter la ville (Blanc, 2010). Le jardin collectif, tout comme l'habitation, constitue pour les jardiniers un élément indispensable de l'habiter urbain, comme en témoignent certains, qui pour rien au monde ne voudrait renoncer à leur jardin, symbole de vie et d'épanouissement. Un quart des 
jardiniers, les jardiniers militants, présentent de plus un discours où la dimension vivrière de la pratique, projetée comme un idéal auquel parvenir dans un univers social renouvelé, est fondamentale. Leur vision du jardinage est celle d'une véritable agronomie pratiquée à grande échelle. Dans ces lieux aux dimensions à la fois récréative, sociale et environnementale, les jardiniers peuvent élaborer, au travers de leur pratique, une véritable réflexion sur les systèmes de production, voire sur l'agriculture dans son ensemble. Ils cultivent chacun leur jardin à leur façon, en fonction de leurs préférences, de leurs aspirations, en rapport avec leur appartenance à une classe socio-culturelle. Mais quelle que soit la nature de l'engagement de ces jardiniers dans le jardinage, celui-ci les met en contact avec le travail de la terre et ses contraintes, et réintègre le fait agricole dans la ville.

L'existence des jardins collectifs urbains et la pratique du jardinage contribuent ainsi à atténuer la coupure entre monde urbain et agricole. Objets de convoitise pour les citadins comme le stigmatise le nombre important des demandes, les jardins collectifs traduisent leur besoin de contact avec la terre, davantage que leur besoin de produire pour se nourrir. Ils leur donnent l'opportunité de développer une véritable expérience agricole au sein même de la ville. Si une ville durable peut être considérée comme une ville se maintenant dans le temps, offrant une qualité de vie dans un esprit de mixité sociale et se développant dans un projet politique et collectif (Emelianoff, 2002), les jardins collectifs en sont bien des éléments. Le soutien à la pratique du jardinage dans les villes par les politiques publiques semble donc pouvoir relever d'un véritable enjeu dans la construction de la ville durable et fertile. Le jardin collectif, en particulier familial, a depuis son origine une fonction nourricière et sociale; il a contribué à améliorer les conditions de vie des citadins et poursuit encore aujourd'hui cet objectif. S'il reste une extension de la sphère domestique dans laquelle ce dernier trouve à satisfaire ses besoins de loisir, le modèle du jardin ne cesse d'évoluer; plus qu'un lieu de loisir et de production, de retour à la nature, de convivialité, il devient un lieu d'innovation agronomique, où le jardinier expérimente de nouvelles techniques de production respectueuses de l'environnement. Dans ces lieux d'interface entre ville, nature et agriculture, où l'agricole vient dialoguer avec l'urbain sous des formes plurielles, le citadin redevient acteur dans l'acte de production.

Espaces publics, lieux de production multifonctionnels cultivés selon des principes agroécologiques, les jardins collectifs urbains pourraient porter en germe les ferments d'une nouvelle relation des citadins à l'agriculture, d'une nouvelle urbanité réconciliant ville et agriculture. Alors que les municipalités, de même que les institutions de gestion des jardins familiaux (Consales, 2008), promeuvent auprès des jardiniers la mise en œuvre de pratiques respectueuses de l'environnement, ces résultats sur les pratiques agronomiques des jardiniers montpelliérains suggèrent qu'ils pourraient être des lieux privilégiés de diffusion d'une culture de l'agro-écologie à l'échelle urbaine. 


\section{BIBLIOGRAPHIE}

Barthel S., Parker J., Ernstson H., 2013, "Food and Green Space in Cities: A Resilience Lens on Gardens and Urban Environmental Movements", Urban studies, http://usj.sagepub.com/content/ early/2013/01/28/0042098012472744.abstract, DOI : 10.1177/0042098012472744.

Blanc N., 2010, « L'habitabilité urbaine ». in Coutard O. et Levy J.P. (dir.), Écologies urbaines, Paris, Economica, p. 169-183.

Bourdeau-Lepage L., Vidal R., 2013, "Comprendre la demande sociale de nature en ville », in Chomarat C. (dir.), Nature urbaine en projet, Archibooks, collection Crossborders, chapitre 2, p. 26-38.

Cabedoce C. et Pierson P., 1996, Cent ans d'histoire des jardins ouvriers : 1896-1996, La Ligue française du Coin de Terre et du Foyer, Éditions Creaphis.

Cerezuelle D., Roustang G., 2010, « Les jardins familiaux de développement social », in Cerezuelle D., Roustang G. (dir.), L'autoproduction accompagnée. Un levier de changement, Éditions Érès, chapitre 2, p. 45-61.

Consales J.-N., 2003, « Les jardins familiaux de Marseille, Gênes et Barcelone entre enjeux potentiels et fonctions réelles de l'agriculture urbaine ", Rives méditerranéennes, vol. 15, p. 1-11.

Consales J.-N., 2004, Les jardins familiaux à Marseille, Gênes et Barcelone : laboratoires territoriaux de l'agriculture urbaine dans l'Arc Méditerranéen, Thèse de Doctorat, Géographie, université AixMarseille 1.

Consales, 2008. « Jardins familiaux et développement durable : entre discours théoriques et actes concrets ", in Da Lage A., Amat J.-P., Frérot A.-M., Guichard-Anguis S., Julien-Laferrière B., Wicherek S.-P. (dir.), L'après développement durable, Ellipses, p. 23-211.

Corrigan M. P., 2011, "Growing what you eat: Developing community gardens in Baltimore, Maryland", Applied Geography, vol. 31, n 4, p. 1232-1241.

Bouvier-Daclon N., Sénécal G., 2001, « Les jardins communautaires de Montréal : un espace social ambigu ", Loisirs et société., vol. 24, n² 2, p. 507-531.

http://www.erudit.org/revue/ls/2001/v24/n2/000193ar.html?vue=resume

Dubost F., 1997, Les jardins ordinaires, Paris, L'harmattan, 174 p.

Duchemin E., Wegmuller F., Legault M., 2010, « Agriculture urbaine : un outil multidimensionnel pour le développement des quartiers, VertigO, vol. 10, n², http://vertigo.revues.org/10436, DOI : 10.4000/vertigo.10436.

Duchemin E., 2011, « Montréal, porte d'entrée des jardins partagés en terres francophones, $L a$ revue durable, vol. 43, p. 44-46.

Duchemin E., 2013, « Multifonctionnalité de l'agriculture urbaine : perspective de chercheurs et de jardiniers ", in Duchemin E. (dir.), Agriculture urbaine : aménager et nourrir la ville, chapitre 2, Éditions Vertigo, p. 97-107.

Emelianoff C., 2002, «La notion de ville durable dans le contexte européen. Quelques éléments de cadrage ", Cahiers français, Enjeux et politiques de l'environnement, vol. 306, p. 28-35. 
Francis, C., Lieblein, G., Gliessman, S., Breland, T. A., Creamer, N., Harwood, R., Salomonsson, L., Helenius, J., Rickerl, D., Salvador, R., Wiedenhoeft, M., Simmons, S., Allen, P., Altieri, M., Flora, C., et Poincelot, R., 2003, "Agroecology: The ecology of food systems", Journal of Sustainable Agriculture, vol. 22, n 3, p. 99-118.

Grandchamp-Florentino L., 2012, "L'agriculture urbaine, enjeu de la ville durable ", Revue des Sciences Sociales, Université de Strasbourg, vol. 47, La ville aux défis de l'environnement, p. 140-150.

Guitard D., Pickering C., Byrne J., 2012, «Past results and future directions in urban community gardens research », Urban forestry and urban greening, vol. 11. $\mathrm{n}^{\circ}$ 4. p. 364-373., http:// www.academia.edu/2088440/ Past_results_and_future_directions_in_urban_community_gardens_research.

Guyon F., 2005, « Les jardins familiaux : miroirs des politiques de la cité », Loisir et société, vol. 27, p. 529-546.

Guyon F., 2008, «Les jardins familiaux aujourd'hui : des espaces socialement modulés », Espaces et sociétés, vol. 3, n 134, p. 131-147.

Kurtz H.E., 2001. "Differentiating multiple meanings of gardened community », Urban Geography, vol. $2, \mathrm{n}^{\circ} 7$, p. 656-670.

McClintock N., 2010, « Why farm the city? Theorizing urban agriculture through a lens of metabolic rift », Cambridge Journal of regions, economy and society, vol. 3, p. 191-207.

Moinar M., 2013, Les rapports à la nature et les modes d'habiter à travers les jardins ouvriers et familiaux en France et en Hongrie, analyse et comparaison, Thèse de Doctorat, Géographie, Université Paris 1.

Monediaire G., 1999, Agricultures urbaines et ville durable européenne : droits et politiques du jardinage familial urbain en Europe, Limoges, Presses Universitaires de Limoges, 336 p.

Ottmann M. M. A., Maantay J. A., Grady K., Fonte N., 2012, "Characterization of urban agricultural practices and gardeners' perceptions in Bronx community gardens, New York City", Cities and the environment (CATE),vol. 5, http://digitalcommons.lmu.edu/cate/vol5/iss1/13.

Paddeu F., 2012, «L'agriculture urbaine dans les quartiers défavorisés de la métropole NewYorkaise : la justice alimentaire à l'épreuve de la justice sociale ", Vertigo - la revue électronique en sciences de l'environnement, vol. 3, n 2, http://vertigo.revues.org/12686, DOI : 10.4000/ vertigo.12686.

Pashchenko O., Consales J. N., 2010, « Les jardins collectifs : derrière une seule notion, des réalités territoriales contrastées ", Durabilis, magazine expérientiel de l'environnement et du développement durable, http://www.magazine-durabilis.com/les-jardins-collectifs-des-realites-territorialescontrastees/4/.

Pereira I., 2009, « Les jardins partagés, un exemple d'entraide libertaire. Entretien avec L. Baudelet ", Réfractions, vol. 23, p. 93-103.

Pourrias J., Boitard M., Daniel A. C., Aubry C., 2013, « La fonction alimentaire des jardins associatifs en question? », Pour, vol. 215/216, Dossier alimentation et territoire, p. 333-347.

Pudup M. B., 2008, "It takes a garden : cultivating citizen subjects in organized garden projects", Geoforum, vol. 39, p. 1228-1240.

Quellier F., 2012. Histoire du jardin potager, Armand Colin, 191 p.

Reyburn S., 2002, « Le cadre de vie et les jardins potagers communautaires à Montréal », VertigO la revue électronique en sciences de l'environnement, vol. 12, $\mathrm{n}^{\circ}$ 2, http://vertigo.revues.org/3794, DOI : $10.4000 /$ vertigo.3794. 
Reyburn S., 2006, Évaluation de la contribution de l'agriculture urbaine communautaire montréalaise à l'amélioration du cadre de vie, Thèse pour l'obtention du grade de Philosophiae doctor en études urbaines, Montréal, Urbanisation, culture et société, université du Québec à Montréal, 229 p. http://www.ucs.inrs.ca/sites/default/files/ReyburnStefanPhD2006.pdf.

Salomon Cavin J. 2012, «Entre ville stérile et ville fertile, l'émergence de l'agriculture urbaine en Suisse », Environnement urbain/Urban Environment, vol. 6, p. 17-31.

Scheromm P., 2013, « Les jardins collectifs, entre nature et agriculture », Métropolitiques, http:// www.metropolitiques.eu/Les-jardins-collectifs-entre.html.

Shepard B., 2014, "Community gardens, creative community organizing and environmental activism", in Gray M. Coates J., Hetherington T. (dir.), Environmental social work, Abingdon, Oxon, Routledge, p. 142-156.

Smith V. M., Greene R.B., Silbernagel J., 2013, "The social and spatial dynamics of community food production : a landscape approach to policy and program development", Landscape ecology in practice, http://link.springer.com/article/10.1007/s10980-013-9891-z.

Soulard C. T., 1999, Les agriculteurs et la pollution des eaux, proposition d'une géographie des pratiques, Thèse de Doctorat, Géographie, Université Paris 1 Panthéon-Sorbonne, CNRS-LADYSS, INRA-SAD, p. $122-155$.

Vitiello D., Wolf-Powers L., 2014, "Growing food to grow cities? The potential of agriculture for economic and community development in the United States", Community Developement Journal, http://cdj.xfordjournals.org/, DOI: 10.1093/cdj/bst087.

Weber F., 1998, L'honneur des jardiniers. Les potagers dans la France du XX ${ }^{e}$ siècle, Belin, 224 p.

Weber M., 1951, Essai sur la théorie de la science, 1904-1917, traduction partielle par J. Freund, Plon,

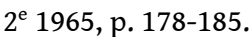

Wegmuller F., Duchemin E., 2010, « Multifonctionnalité de l'agriculture urbaine à Montréal : étude des discours au sein du programme des jardins communautaires ", Vertigo - la revue électronique en sciences de l'environnement, vol. 10, $\mathrm{n}^{\circ} 2$, http://vertigo.revues.org/10445, DOI : 10.4000 /vertigo. 10445 .

\section{NOTES}

1. Définition issue de la proposition de loi relative aux jardins collectifs adoptée par le sénat et retransmise à l'assemblée nationale le 2 juillet 2012.

2. http://jardins-partages.org/telechargezmoi_files/Lille97.pdf.

3. http://jardins-partages.org/.

\section{RÉSUMÉS}

Les jardins collectifs se multiplient aujourd'hui dans les villes occidentales. À Montpellier, leur essor est lié à la demande des citadins et soutenu par la municipalité. Quarante entretiens semi- 
directifs ont permis d'identifier les motivations des jardiniers, leurs pratiques agronomiques et leur conception du jardinage et de l'agriculture. Dans ces jardins collectifs, lieux aux fonctions plurielles, une reconnexion des citadins avec l'agriculture s'opère, même si l'objectif de production alimentaire n'y est pas affiché comme une priorité. Cette nature urbaine multifacettes, promue par les citadins et qui intéresse les politiques urbaines, ne porte-t-elle pas en germe l'amorce d'une nouvelle relation entre ville et agriculture?

Collective gardens, have multiplied in developed countries. In Montpellier city, their boost is due to the request of the citizens and supported by the municipality. Forty semi-structured interviews were conducted to investigate gardeners' motivations, agricultural practices and view of gardening and farming. Reconnection of dwellers with farming was identified in these places with multiple functions, even if the objective of food production is not a priority. Our results suggest that this cultivated ordinary nature promoted by the city dwellers carries a new link between cities and agriculture.

\section{INDEX}

Keywords : collective gardens, city, agricultural practices, motivations, dwellers

Mots-clés : jardins collectifs, ville, pratiques agronomiques, motivations, citadins

\section{AUTEUR}

\section{PASCALE SCHEROMM}

Pascale Scheromm est géographe, ingénieur de recherche à l'INRA, elle développe depuis deux ans un programme de recherche sur les espaces et les pratiques de l'agriculture intra-urbaine au sein de l'équipe Innovations territoriales de l'UMR INRA/Cirad/SupAgro Innovation dans l'agriculture et l'agroalimentaire, scheromm@supagro.inra.fr 